\title{
Pacific
}

Journal of

Mathematics

MEAN-VALUE CHARACTERIZATION OF PLURIHARMONIC AND SEPARATELY HARMONIC FUNCTIONS

LEV ABRAMOVICH AǏZENBERG, CARLOS A. BERENSTEIN AND L. WeRTHEIM 


\section{MEAN-VALUE CHARACTERIZATION OF PLURIHARMONIC AND SEPARATELY HARMONIC FUNCTIONS}

\section{L.A. Aizenberg C.A. Berenstein And L. Wertheim}

We show that separately harmonic functions and pluriharmonic functions in $\mathrm{C}^{n}$ can be characterized by a finite number of mean-value conditions over boundaries of ellipsoids or distinguished boundaries of polydisks. This is a generalization of the Delsarte-Lions characterization of harmonic functions and of the Morera theorem for holomorphic functions.

\section{Introduction.}

Let us recall the converse of Gauss's mean-value theorem as proved by Delsarte and Lions [11], it says that for any $n \geq 2$ there is a finite set $H_{n}$, $1 \in H_{n}$, such that if $r_{1}>0, r_{2}>0$ and $r_{1} / r_{2} \notin H_{n}$ then any $f \in C\left(\mathbf{R}^{n}\right)$ satisfying

$$
\int_{S\left(x, r_{j}\right)} f(y) d \sigma_{j}(y)=f(x), \quad\left(j=1,2 ; x \in \mathbf{R}^{n}\right)
$$

is harmonic in $\mathbf{R}^{n}$. Here $S\left(x, r_{j}\right)$ is the sphere of center $x$ and radius $r_{j}$, $d \sigma_{j}$ is the normalized Lebesgue measure on the sphere.In fact, $H_{3}=\{1\}$, so that any two distinct radii are sufficient in dimension 3. In [9] this result is extended to arbitrary non-compact irreducible symmetric spaces of rank 1 . Finally, from $[\mathbf{7}, \mathbf{8}]$ we conclude that in $\mathbf{R}^{n}$ there is a local version of this theorem (in fact, this result extends to symmetric spaces of rank 1), namely if $f \in C(B(0, R)), r_{1}+r_{2}<R$, and satisfies (1), in the sense that (1) holds as long as $|x|+r_{j}<R$, then $f$ is harmonic in the ball $B(0, R)$ of center 0 and radius $R$. On the other hand, we do not have such a satisfactory situation for symmetric spaces of higher rank or the Heisenberg group (or other nilpotent Lie groups for that matter). In the latter case, the results in $[\mathbf{1}, \mathbf{2}]$ are established for $L^{p}$-functions $f$. The reason for this difficulty is slightly different in these two cases. In the case of symmetric spaces $X$ of rank bigger than one it is due to the failure of the Spectral Synthesis Theorem in $C^{\infty}(X)$. [6], while in the Heisenberg group $\mathbf{H}^{n}$ we do not have yet a resonable effective method to study this kind of problem in $C^{\infty}\left(\mathbf{H}^{n}\right)$. In this paper we study 
the characterization of separately harmonic and pluriharmonic functions in $\mathbf{C}^{n}(n \geq 2)$ by means of mean-value properties. That is, by identities of the type (1). As it will become clear later on, we have really a problem of the type that arises in symmetric spaces of rank $\geq 2$. For a general perspective on this kind of problems we refer to $[\mathbf{1 3}, \mathbf{1 4}]$.

This research was carried while the first author held a visiting Professorship at the University of Maryland. He would like to thank the Mathematics Department and the Institute for Systems Research for their hospitality.

\section{Necessary conditions.}

From now on we will be working in $\mathbf{C}^{n}$ with $n \geq 2$, the orientation of the space is the usual one that makes the differential form

$$
\frac{1}{(2 i)^{n}} d \bar{z}_{1} \wedge d z_{1} \wedge \cdots \wedge d \bar{z}_{n} \wedge d z_{n}>0
$$

We also recall that this form equals

$$
\frac{(-1)^{\frac{n(n-1)}{2}}}{(2 i)^{n}} d \bar{z} \wedge d z:=\frac{(-1)^{\frac{n(n-1)}{2}}}{(2 i)^{n}} d \bar{z}_{1} \wedge \cdots \wedge d \bar{z}_{n} \wedge d z_{1} \wedge \cdots \wedge d z_{n} .
$$

The notation $d \bar{\zeta}[k]=d \bar{\zeta}_{1} \wedge \ldots \wedge d \bar{\zeta}_{k-1} \wedge d \bar{\zeta}_{k+1} \wedge \ldots \wedge d \bar{\zeta}_{n}$ will be used throughout.

Let us also introduce the following differential form

$$
\nu(\zeta-a)=(-1)^{\frac{n(n-1)}{2}} \frac{1}{(2 \pi i)^{n}} \sum_{k=1}^{n}(-1)^{k-1}\left(\bar{\zeta}_{k}-\bar{a}_{k}\right) d \bar{\zeta}[k] \wedge d \zeta .
$$

A domain $D \subseteq \mathbf{C}^{n}$ is called $n$-circular (or Reinhardt domain) with center at the point $a$, if $z \in D$ implies $\left(a_{1}+\left(z_{1}-a_{1}\right) e^{i t_{1}}, \ldots, a_{n}+\left(z_{n}-a_{n}\right) e^{i t_{n}}\right) \in D$ for $0 \leq t_{j} \leq 2 \pi, j=1,2, \ldots, n$. Such a domain is called complete, if with each point $z^{0} \in D, D$ contains the whole polydisk $Q_{r}(a)=\left\{z:\left|z_{j}-a_{j}\right|<r_{j}, j=\right.$ $1, \ldots, n\}$, where $r_{j}=\left|z_{j}^{0}-a_{j}\right|, j=1, \ldots, n$. Henceforth the space $C(D)$ shall be the space of real valued continuous functions in $D$.

Theorem 2.1. If $D \subset \mathbf{C}^{n}$ is a complete bounded $n$-circular domain with center at the point $a$ and $f$ is a separately harmonic function in $D$ (i.e., harmonic with respect to each variable $\left.z_{j}, j=1, \ldots, n\right)$ and continuous in $\bar{D}$, then

$$
\frac{\pi^{n}}{n \operatorname{vol}(D)} \int_{\partial D} f(\zeta) \nu(\zeta-a)=f(a)
$$

Also, if $Q_{r}=Q_{r}(a)$ is a polydisk with center $a$, then for any function $f$, separately harmonic in $Q_{r}$ and continuous in $\bar{Q}_{r}$ one has

$$
\frac{1}{(2 \pi)^{n} r_{1} \ldots r_{n}} \int_{\Delta_{r}(a)} f(\zeta)\left|d \zeta_{1}\right| \wedge \ldots \wedge\left|d \zeta_{n}\right|=f(a)
$$


where $\Delta_{r}(a)$ is the distinguished boundary of the polydisk $Q_{r}(a)$, i.e., $\Delta_{r}(a)=$ $\left\{z:\left|z_{j}-a_{j}\right|=r_{j}, j=1, \ldots, n\right\}$.

Proof. The equality (3) is obtained using that any function separately harmonic in $Q_{r}$ and continuous in $\bar{Q}_{r}$ satisfies the mean value property with respect to integration over the distinguished boundary $\Delta_{r}$. In order to prove formula (2) we use the following lemma, whose proof is immediate.

Lemma 2.2. Let $R=\left(\left|\zeta_{1}-a_{1}\right|^{2}, \ldots,\left|\zeta_{n}-a_{n}\right|^{2}\right)=\left(R_{1}, \ldots, R_{n}\right)$. The form $\nu$ satisfies the following identity

$$
\nu(\zeta-a)=\frac{1}{(2 \pi i)^{n}} \mu(R) \wedge \frac{d \zeta}{\zeta-a}
$$

where

$$
\frac{d \zeta}{\zeta-a}=\frac{d \zeta_{1}}{\zeta_{1}-a_{1}} \wedge \ldots \wedge \frac{d \zeta_{n}}{\zeta_{n}-a_{n}}
$$

and

$$
\mu(R)=(-1)^{\frac{n(n-1)}{2}} \sum_{k=1}^{n}(-1)^{k-1} R_{k} d R[k] .
$$

It follows now from (4) and the identity (3) that the formula (2) must be correct up to a multiplicative constant. This constant can be computed using $f \equiv 1$ and applying the Stokes formula:

$$
\int_{\partial D} \nu(z-a)=\frac{(-1)^{\frac{n(n-1)}{2}} n}{(2 \pi i)^{n}} \int_{D} d \bar{z} \wedge d z=\frac{n}{\pi^{n}} \operatorname{vol}(D) .
$$

This concludes the proof.

Let us recall that a domain $D_{1} \subset \mathbf{C}^{n}$ is called circular or Cartan domain with center at the point $a$, if $z \in D_{1}$ implies $(z-a) e^{i t} \in D_{1}$ for $0 \leq t \leq 2 \pi$. Such a domain is said to be complete, if for each each point $z^{0} \in D_{1}, D_{1}$ contains the whole disk $\left\{a_{1}+\left(z_{1}^{0}-a_{1}\right) t, \ldots, a_{n}+\left(z_{n}^{0}-a_{n}\right) t: t \in \mathbf{C},|t|<1\right\}$. An example is the ball $B_{\rho}=\{z:|z-a|<\rho\}$.

Theorem 2.3. If $D_{1} \subset \mathbf{C}^{n}$ is a complete bounded circular domain with center at the point $a$ and $f$ is a pluriharmonic function in $D_{1}$ (i.e., real part of a holomorphic function) and continuous in $\bar{D}_{1}$, then

$$
\frac{\pi^{n}}{n \operatorname{vol}\left(D_{1}\right)} \int_{\partial D_{1}} f(\zeta) \nu(\zeta-a)=f(a) .
$$


If $Q_{r}^{1}$ is a "circular polydisk" with center at a, i.e., $Q_{r}^{1}=Q_{r}^{1}(a)=\{z$ : $\left.\left|b_{j}^{1}\left(z_{1}-a_{1}\right)+\cdots+b_{j}^{n}\left(z_{n}-a_{n}\right)\right|<r_{j}, j=1, \ldots, n\right\}$, det $\left\|b_{j}^{l}\right\| \neq 0$, then for any function $f$ which is pluriharmonic in $Q_{r}^{1}$ and continuous in $\bar{Q}_{r}^{1}$ we have

$$
\begin{aligned}
& f(a)=\frac{1}{(2 \pi)^{n} r_{1} \ldots r_{n}} \int_{\Delta_{r}^{1}(a)} f(\zeta) \mid d\left(b_{1}^{1}\left(\zeta_{1}-a_{1}\right)+\cdots\right. \\
& \left.\quad+b_{1}^{n}\left(\zeta_{n}-a_{n}\right)\right)|\wedge \ldots \wedge| d\left(b_{n}^{1}\left(\zeta_{1}-a_{1}\right)+\cdots+b_{n}^{n}\left(\zeta_{n}-a_{n}\right)\right) \mid
\end{aligned}
$$

where $\Delta_{r}^{1}(a)=\left\{z:\left|b_{j}^{1}\left(z_{1}-a_{1}\right)+\cdots+b_{j}^{n}\left(z_{n}-a_{n}\right)\right|=r_{j}, j=1, \ldots, n\right\}$ is the distinguished boundary of the circular polydisk $Q_{r}^{1}(a)$.

Proof. A pluriharmonic function is obviously separately harmonic as well, therefore formula (3) is valid for it and (6) can be derived from (3) by a linear transformation (a linear transformation conserves pluriharmonicity, because a function is pluriharmonic if and only if its restriction to each complex line is harmonic). The proof of (5) requires the following lemma, whose proof is contained in [4, Lemma 17.8]. All complex lines $\alpha$ passing through $a$ and not lying in the hyperplane $\left\{z: z_{1}=a_{1}\right\}$ can be written in the form

$$
\alpha(v, a)=\left\{z: z_{1}=a_{1}+t, z_{2}=a_{2}+v_{2} t, \ldots, z_{n}=a_{n}+v_{n} t, t \in \mathbf{C}\right\}
$$

where $v=\left(v_{2}, \ldots, v_{n}\right)$. With this notation we have

\section{Lemma 2.4.}

$$
\nu(\zeta-z)=\frac{1}{2 \pi i}|t|^{2 n} \frac{d t}{t} \wedge \frac{(-1)^{n-1}}{(2 \pi i)^{n-1}} d \bar{v} \wedge d v
$$

From this Lemma and the mean-value property for harmonic functions in C we obtain (5) up to a multiplicative constant, which can be computed in the same way as it was done in Theorem 2.1.

Remark 2.5. Lemma 2.4 has appeared in work of Kytmanov (see [5]) and in [12].

Remark 2.6. Formulae (2), (3), (5) and (6) in the particular case $n=1$ are the classical mean value theorem for harmonic functions.

Remark 2.7. It is easy to show that each of the conditions above is suffi= cient for the corresponding class of functions, but we do not prove it here, because stronger statements will be proved below.

\footnotetext{
${ }^{1}$ We are using this terminology and notation to distinguish it from a usual polydisk, which is $n$-circular.
} 
Remark 2.8. It can be shown that in the formulae (2) and (5) the form $\nu$ can be replaced by the Bochner-Martinelli kernel (up to a \pm 1 )

$$
\omega(\zeta-a, \overline{\zeta-a})=\frac{(n-1) !}{|\zeta-a|^{2 n}} \nu(\zeta-a),
$$

except that the constant in front of the integrals in the formulas corresponding to (2) and (5) will be independent of the domain.

In the case of the ball the differential form $\nu$ and the Bochner-Martinelli kernel differ from the surface area element by constants. More generally, one has the following two mean-value results.

Proposition 2.9. If $f$ is separately harmonic in the $n$-circular ellipsoid

$$
\Omega=\left\{z: b_{1}\left|z_{1}-a_{1}\right|^{2}+\cdots+b_{n}\left|z_{n}-a_{n}\right|^{2}<r^{2}\right\}
$$

and continuous in $\bar{\Omega}$, then

$$
\frac{1}{A(\partial \Omega)} \int_{\partial \Omega} f(\zeta) d s(\zeta)=f(a)
$$

Proof. For the proof we can assume $a=0$. Then, observe that $\partial \Omega$ and the area measure are invariant under the action of the group $G=S^{1} \times \cdots \times S^{1}$, whose normalized Haar measure $d \mu$ is $(2 \pi)^{-n} d \theta_{1} \cdots d \theta_{n}$. As $f$ is separately harmonic, if $\left(\zeta_{1}^{0}, \ldots, \zeta_{n}^{0}\right)$ is a fixed point of $\partial \Omega$ then

$$
\int_{G} f\left(e^{i \theta_{1}} \zeta_{1}^{0}, \ldots, e^{i \theta_{n}} \zeta_{n}^{0}\right) d \mu(\theta)=f(0) .
$$

Therefore, replacing $\zeta$ by $e^{i \theta} \cdot \zeta$ (with the obvious meaning of this product) in the left-hand side of (8) and integrating on $G$ against the measure $d \mu$, we obtain the desired identity.

Proposition 2.10. If $f$ is pluriharmonic in the circular ellipsoid

$$
\Omega_{1}=\left\{z: \sum_{j=1}^{n} b_{j}\left|c_{j}^{1}\left(z_{1}-a_{1}\right)+\cdots+c_{j}^{n}\left(z_{n}-a_{n}\right)\right|^{2}<r^{2}\right\}
$$

and continuous in $\bar{\Omega}_{1}$, where $\operatorname{det}\left\|c_{j}^{k}\right\| \neq 0$, then

$$
\frac{1}{A\left(\partial \Omega_{1}\right)} \int_{\partial \Omega_{1}} f(\zeta) d s(\zeta)=f(a) .
$$

Proof. The proof is similar, just use that pluriharmonicity is invariant under linear invertible transformations. 


\section{Sufficient conditions in terms of the mean-value property for the whole boundary.}

Let us recall that the Fourier transform of the normalized area measure of the unit ball in $\mathbf{C}^{n}$ is given by $j_{n-1}\left(\left(z_{1}^{2}+\cdots+z_{n}^{2}\right)^{\frac{1}{2}}\right)$, where, in terms of Bessel functions we have

$$
j_{n-1}(\zeta)=\Gamma(n)\left(\frac{2}{\zeta}\right)^{n-1} J_{n-1}(\zeta)
$$

The set $H_{2 n}$ of the introduction is the set of positive quotients of zeros of $j_{n-1}(\zeta)-1$. (For $\mathbf{R}^{m}$ just replace $n=m / 2$.) In this section we will consider mean-value properties with respect the following $n$-circular ellipsoids with center at the point $a$ :

$$
D_{j, k}(a)=\left\{z: b_{j}^{1}\left|z_{1}-a_{1}\right|^{2}+\cdots+b_{j}^{n}\left|z_{n}-a_{n}\right|^{2}<r_{j, k}^{2}\right\}
$$

where $k=1,2, j=1, \ldots, n$, all $b_{j}^{l}>0$.

Theorem 3.1. Let $f \in C\left(\mathbf{C}^{n}\right)$ be such that for each $a \in \mathbf{C}^{n}$ the $2 n$ conditions obtained by setting in (2) $D=D_{j, k}(a), j=1, \ldots, n$ and $k=1,2$, hold. If no $r_{j, 1} / r_{j, 2}$ belongs to $H_{2 n}$, and

$$
\operatorname{det}\left\|1 / b_{j}^{l}\right\| \neq 0
$$

then $f$ is separately harmonic in $\mathbf{C}^{n}$.

Proof. Fixing $j$, consider the change of variables $w_{l}=\sqrt{b_{j}^{l}} z_{l}$. Then the family of ellipsoids $D_{j, k}(a)$ is transformed to a family of balls $B\left(\tilde{a}, r_{j, k}\right), \tilde{a}_{l}=\sqrt{b_{j}^{l}} a_{l}$. The function $\tilde{f}\left(w_{1}, \ldots, w_{n}\right)=f\left(z_{1}, \ldots, z_{n}\right)=f\left(w_{1} / \sqrt{b_{j}^{1}}, \ldots, w_{n} / \sqrt{b_{j}^{n}}\right)$ satisfies the following condition

$$
\frac{\pi^{n}}{n \operatorname{vol}\left(B\left(\tilde{a}, r_{j, k}\right)\right)} \int_{\partial B\left(\tilde{a}, r_{j, k}\right)} \tilde{f}\left(w_{1}, \ldots, w_{n}\right) \nu(w-\tilde{a})=\tilde{f}(\tilde{a}) .
$$

Using the notation $w-\tilde{a}=\xi+i \eta$ it can be shown that (see [5, p. 24]) $\operatorname{Re} \nu(w-\tilde{a})=\frac{(-1)^{\frac{n(n-1)}{2}}}{2 \pi^{n}} \sum_{j=1}^{n}\left((-1)^{n-1} \xi_{j} d \xi[j] \wedge d \eta+(-1)^{n+j-1} \eta_{j} d \xi \wedge d \eta[j]\right)$.

In other words, in the integral we are averaging $\tilde{f}$ with respect to the volume form on the sphere $\partial B\left(\tilde{a}, r_{j, k}\right)$. According to the Delsarte-Lions mean-value 
characterization of harmonic functions [11], $\tilde{f}$ is harmonic, i.e., for each $z \in \mathbf{C}^{n}$

$$
0=\sum_{l=1}^{n} \frac{\partial^{2}}{\partial \bar{z}_{l} \partial z_{l}} f\left(z_{1} / \sqrt{b_{j}^{1}}, \ldots, z_{n} / \sqrt{b_{j}^{n}}\right)=\sum_{l=1}^{n} \frac{1}{b_{j}^{l}} \frac{\partial^{2} f(\tilde{z})}{\partial \tilde{\bar{z}}_{l} \partial \tilde{z}_{l}}
$$

where $\tilde{z}=\left(\sqrt{b_{j}^{1}} z_{1}, \ldots, \sqrt{b_{j}^{n}} z_{n}\right)$. Because $z$ is arbitrary, $\tilde{z}$ is arbitrary as well. In other words, for all $z \in \mathbf{C}^{n}$

$$
\sum_{l=1}^{n} \frac{1}{b_{j}^{l}} \frac{\partial^{2} f(z)}{\partial \bar{z}_{l} \partial z_{l}}=0
$$

In particular, $f$ is real analytic. Since (11) holds for every $j=1, \ldots, n$, it follows from (10) that

$$
\frac{\partial^{2} f(z)}{\partial \bar{z}_{l} \partial z_{l}}=0, \quad l=1, \ldots, n
$$

i.e., $f$ is separately harmonic in $\mathbf{C}^{n}$.

We have already mentioned that there is a local version of the DelsarteLions two-radii theorem, the same argument in [8] shows that the same is true for the two-radii theorem we used in the last proof. This is the reason of the first condition imposed below.

Theorem 3.2. Let a domain $\Omega$ be the union of ellipsoids $\left\{z: b_{1}^{1}\left|z_{1}-a_{1}\right|^{2}+\right.$ $\left.\cdots+b_{1}^{n}\left|z_{n}-a_{n}\right|^{2}<r^{2}\right\}$ of radii $r$ strictly bigger than $r_{1,1}+r_{1,2}$. Let $f \in C(\Omega)$ and assume that for each point $a \in \Omega$ such that $D_{r_{1, k}}(a) \subset \Omega$ ( $k$ either 1 or 2) the mean value condition holds (2) holds, where $D=D_{r_{1, k}}(a)$, with the corresponding value of $k$. Assume further that for each $2 \leq j \leq n$ there is a closed ellipsoid $E_{j}$ of the form $\left\{z: b_{j}^{1}\left|z_{1}-a_{j, 1}\right|^{2}+\cdots+b_{j}^{n}\left|z_{n}-a_{j, n}\right|^{2} \leq\right.$ $\left.\left(r_{j, 1}+r_{j, 2}\right)^{2}\right\}$ contained in $\Omega$ (for a convenient choice of the point $\left.a_{j}\right)$. If the corresponding pair of mean value conditions (2) hold for all $D_{r_{j, k}}(a) \subset E_{j}$ then $f$ is separately harmonic in $\Omega$.

Proof. The first part of the proof of Theorem 3.1, the geometric condition on $\Omega$, and the possibility to localize the two-radii theorem allows us to conclude $f$ satisfies (11) for $j=1$ in $\Omega$, and thus it is real analytic everywhere. The other conditions guarantee that $f$ satisfies the $j$-th condition (11) in a neighborhood of the point $a_{j}$. It now follows from the connectedness of $\Omega$ and the real analyticity of $f$ that all the conditions (11) are satisfied everywhere. Therefore, $f$ is separately harmonic in $\Omega$.

Remark 3.3. Theorems 3.1 and 3.2 demand that the identity (2) must hold for $n$ pairs of ellipsoids. These theorems are not true if we impose 
conditions on only $(n-1)$ pairs. In fact, if we had only $(n-1)$ pairs, we would obtain a system of equations like (11), but consisting only of $(n-1)$ equations. Such a system has a nontrivial solution

$$
\frac{\partial^{2} f(z)}{\partial \bar{z}_{l} \partial z_{l}}=c_{l}, \quad l=1, \ldots, n
$$

where some $c_{l} \neq 0$. Any solution of the system (12) satisfies our conditions for $(n-1)$ pairs of ellipsoids, but it is not separately harmonic.

Consider now the following circular ellipsoids with center at the point $a$ :

$$
\begin{gathered}
D_{j, k}^{p}(a)=\left\{z: \sum_{l=1}^{n} b_{j}^{l}\left|c_{l 1}^{p}\left(z_{1}-a_{1}\right)+\cdots+c_{l n}^{p}\left(z_{n}-a_{n}\right)\right|^{2}<r_{j, k}^{2}\right\}, \\
b_{j}^{l}>0 ; j=1, \ldots, n ; k=1,2 ; p=1, \ldots, n .
\end{gathered}
$$

Let $\left\|d_{l m}^{p}\right\|,(l, m=1, \ldots, n)$ be the inverse matrix of $\left\|c_{l m}^{p}\right\|$ for $p$ fixed. Let $Q=\left\|q_{p s, k l}\right\|,(p, s=1, \ldots, n ; k, l=1, \ldots, n)$ be the $n^{2} \times n^{2}$ matrix with the following entries

$$
q_{p s, k l}=d_{k s}^{p} \bar{d}_{l s}^{p}
$$

Theorem 3.4. Let $f \in C\left(\mathbf{C}^{n}\right)$ so that for every $a \in \mathbf{C}^{n}$ the conditions (5) hold for $D_{1}=D_{j, k}^{p}(a), j=1, \ldots, n ; k=1,2 ; p=1, \ldots, n$ ( $2 n$ conditions $)$. If $r_{j, 1}$ and $r_{j, 2}$ are chosen as in Theorem 3.1 , $\operatorname{det}\left\|1 / b_{j}^{l}\right\| \neq 0$, and $\operatorname{det} Q \neq 0$, then $f$ is pluriharmonic.

Proof. Fixing $p$, we can consider $D_{j, k}^{p}(a)$ as an affine image of $D_{j, k}(0)$, where the matrix of the linear map is $\left\|d_{l m}^{p}\right\|$. From the condition (5) and the reasoning preceding Corollaries 2.9 and 2.10, we conclude that the function

$$
\left(w_{1}, \ldots, w_{n}\right) \mapsto f\left(d_{11}^{p} w_{1}+\cdots+d_{1 n}^{p} w_{n}, \ldots, d_{n 1}^{p} w_{1}+\cdots+d_{n n}^{p} w_{n}\right)
$$

satisfies all the conditions of the Theorem 3.1 and, therefore, it is separately harmonic. Hence taking derivatives, we obtain

$$
\sum_{k, l=1}^{n} d_{k s}^{p} \bar{d}_{l s}^{p} \frac{\partial^{2} f}{\partial z_{k} \partial \bar{z}_{l}} \equiv 0
$$

Since $\operatorname{det} Q \neq 0$, we get

$$
\frac{\partial^{2} f}{\partial z_{k} \partial \bar{z}_{l}} \equiv 0, \quad k, l=1, \ldots, n .
$$

This means that $f$ is pluriharmonic. 
Remark 3.5. In this theorem we are considering images of the family of ellipsoids from the Theorem 3.1 under $n$ complex linear transformations. The only restriction on them is the condition $\operatorname{det} Q \neq 0$. In particular, no more than one of these transformations can be unitary. In the case $n=2$ and one of them is the identity (say when $p=1$ ), the condition on the other is :

$$
\operatorname{Im}\left(d_{11}^{2} \bar{d}_{12}^{2} \bar{d}_{21}^{2} d_{22}^{2}\right) \neq 0 \text {. }
$$

Remark 3.6. Theorems $3.1,3.2$ and 3.4 can be rewritten using formulas (8) and (9).

We leave to the reader the statement of local theorems corresponding to Theorem 3.2.

\section{Three circles theorem on the plane.}

Theorem 4.1. Let $\phi$ be a continuous function on C. Suppose that

$$
\lambda\left(\phi, z, r_{1}\right)=\lambda\left(\phi, z, r_{2}\right)=\lambda\left(\phi, z, r_{3}\right), z \in \mathbf{C}
$$

where $\lambda(\phi, z, r)=\frac{1}{2 \pi} \int_{0}^{2 \pi} \phi\left(z+r e^{i \theta}\right) d \theta$. If $\omega=0$ is the only solution of the system

$$
J_{0}\left(r_{1} \omega\right)=J_{0}\left(r_{2} \omega\right)=J_{0}\left(r_{3} \omega\right)
$$

then $\phi$ is harmonic.

Proof. Clearly, $\lambda(\phi, z, r)=\phi * \chi_{r}(z)$, where $\chi_{r} \in \mathcal{E}^{\prime}\left(\mathbf{R}^{2}\right)$ is the following distribution of compact support:

$$
\psi \mapsto \frac{1}{2 \pi} \int_{0}^{2 \pi} \psi\left(r e^{i \theta}\right) d \theta
$$

Therefore, the condition (14) can be rewritten as

$$
\phi *\left(\chi_{r_{1}}-\chi_{r_{2}}\right)=\phi *\left(\chi_{r_{2}}-\chi_{r_{3}}\right)=0 .
$$

Consider the closed convolution ideal in $\mathcal{E}^{\prime}\left(\mathbf{R}^{2}\right)$ generated by the radial distributions $\chi_{r_{1}}-\chi_{r_{2}}$ and $\chi_{r_{2}}-\chi_{r_{3}}$. Our aim is to show that the Laplace operator $\Delta$ (considered as the distribution $\Delta \delta_{0}$ ) belongs to this ideal. (Because any solution of Laplace equation in the sense of distributions is automatically a harmonic function.) Translating this statement into the space $\widehat{\mathcal{E}^{\prime}\left(\mathbf{R}^{2}\right)}$ of Fourier transforms, we have to prove that $\xi^{2}+\eta^{2}$ belongs to the closed ideal generated by $J_{0}\left(r_{1} \sqrt{\xi^{2}+\eta^{2}}\right)-J_{0}\left(r_{2} \sqrt{\xi^{2}+\eta^{2}}\right)$ and $J_{0}\left(r_{2} \sqrt{\xi^{2}+\eta^{2}}\right)-J_{0}\left(r_{3} \sqrt{\xi^{2}+\eta^{2}}\right)$. 
According to $[\mathbf{1 0}, \mathbf{9}]$, it is enough that $J_{0}\left(r_{1} \omega\right)-J_{0}\left(r_{2} \omega\right)$ and $J_{0}\left(r_{2} \omega\right)-J_{0}\left(r_{3} \omega\right)$ have $\omega=0$ as their only common zero.

Remark 4.2. The correspondent statement for only two radii is false. On the other hand, let us show that for almost every choice of distinct $r_{1}, r_{2}, r_{3}$ our conditions hold. In fact, fixing $r_{2} / r_{1} \neq 1$ we have a discrete set of solutions $J_{0}(w)=J_{0}\left(w r_{2} / r_{1}\right)$ and for each such a $w$, we have a discrete set of exceptional values of $r_{3} / r_{2}$ such that $J_{0}\left(w r_{3} / r_{2}\right)=J_{0}(w)$. Hence, we have at most countable set of exceptional values of $r_{3} / r_{2}$ for each fixed $r_{2} / r_{1} \neq 1$.

\section{Sufficient conditions in terms of the mean-value property for distinguished boundaries.}

Theorem 5.1. Let $r_{1}, r_{2}, r_{3}$ be such that they satisfy the conditions of Theorem 4.1. Let $\rho_{1}, \rho_{2}>0$ such that $\rho_{1} / \rho_{2} \notin H_{2}$. If a continuous function $f \in C\left(\mathbf{C}^{n}\right)$ satisfies

$$
\frac{1}{(2 \pi)^{n}} \int_{[0,2 \pi]^{n}} f\left(a_{1}+R_{1} e^{i t_{1}}, \ldots, a_{n}+R_{n} e^{i t_{n}}\right) d t_{1} \ldots d t_{n}=f(a)
$$

for every $a \in \mathbf{C}^{n}$ and any choice of $R_{l}$, such that

$$
R_{l} \in\left\{r_{1}, r_{2}, r_{3}\right\}, l=1, \ldots, n-1 ; \quad R_{n} \in\left\{\rho_{1}, \rho_{2}\right\}
$$

then $f$ is separately harmonic.

Remark. We are considering $2 \cdot 3^{n-1}$ polydisks at each point.

Proof. We proceed by induction on $n$. When $n=1$ it is Delsarte-Lions's theorem [11].

For $n>1$ consider the auxiliary function

$$
F\left(z_{1}\right)=\frac{1}{(2 \pi)^{n-1}} \int_{[0,2 \pi]^{n-1}} f\left(z_{1}, a_{2}+R_{2} e^{i t_{2}}, \ldots, a_{n}+R_{n} e^{i t_{n}}\right) d t_{2} \ldots d t_{n}
$$

with $a_{2}, \ldots, a_{n}, R_{2}, \ldots, R_{n}$ fixed. By virtue of (14) we have

$$
\lambda\left(F, z_{1}, r_{1}\right)=\lambda\left(F, z_{1}, r_{2}\right)=\lambda\left(F, z_{1}, r_{3}\right)=f\left(z_{1}, a_{2}, \ldots, a_{n}\right), \quad \forall z_{1} \in \mathbf{C} .
$$

Therefore, by Theorem 4.1, $\mathrm{F}$ is harmonic and so

$$
\lambda\left(F, z_{1}, r_{1}\right)=F\left(z_{1}\right)=f\left(z_{1}, a_{2}, \ldots, a_{n}\right) .
$$


Hence, $f$ is harmonic with respect to the first variable. Fixing $z_{1}$ for a moment, consider the function

$$
g\left(z_{2}, \ldots, z_{n}\right)=f\left(z_{1}, z_{2}, \ldots, z_{n}\right) .
$$

From (15) and (17) we observe that $g$ satisfies all the conditions of the theorem. Hence by the induction hypothesis $g$ is separately harmonic with respect to the variables $z_{2}, \ldots, z_{n}$, and thus $f$ is separately harmonic with respect to all variables.

Consider the following circular polydisks

$$
Q_{p}(a ; R)=\left\{z:\left|c_{j 1}^{p}\left(z_{1}-a_{1}\right)+\cdots+c_{j n}^{p}\left(z_{n}-a_{n}\right)\right|<R_{j}, j=1, \ldots, n\right\}
$$

for $p=1, \ldots, n, R_{j} \in\left\{r_{1}, r_{2}, r_{3}\right\}, j=1, \ldots, n-1$, and $R_{n} \in\left\{\rho_{1}, \rho_{2}\right\}$. Furthermore, let

$$
\Delta_{p}(a ; R)=\left\{z:\left|c_{j 1}^{p}\left(z_{1}-a_{1}\right)+\cdots+c_{j n}^{p}\left(z_{n}-a_{n}\right)\right|=R_{j}, j=1, \ldots, n\right\}
$$

be its distinguished boundary. Let $Q$ be the same matrix as in Theorem 3.3.

Theorem 5.2. Let $r_{1}, r_{2}, r_{3}, \rho_{1}, \rho_{2}$ be as in Theorem 5.1, $\operatorname{det} Q \neq 0$. Let $f \in C\left(\mathbf{C}^{n}\right)$ be such that for any $a \in \mathbf{C}^{n}, p=1, \ldots, n$ and any posible choices of $R_{j}$ we have

$$
\begin{array}{r}
f(a)=\frac{1}{(2 \pi)^{n} R_{1} \ldots R_{n}} \int_{\Delta_{p}(a ; R)} f(\zeta)\left|d\left(c_{11}^{p}\left(\zeta_{1}-a_{1}\right)+\cdots+c_{1 n}^{p}\left(\zeta_{n}-a_{n}\right)\right)\right| \wedge \\
\ldots \wedge\left|d\left(c_{n 1}^{p}\left(\zeta_{1}-a_{1}\right)+\cdots+c_{n n}^{p}\left(\zeta_{n}-a_{n}\right)\right)\right|
\end{array}
$$

Then $f$ is pluriharmonic.

Proof. It is based on Theorem 5.1 and a reasoning similar to that in Theorem 3.3 , so we omit the details.

Remark 5.3. A natural question is whether it is possible to diminish the number of polydisks in Theorems 5.1 and 5.2. There is a reasonable expectation to obtain an analogous to the Theorem 5.1 result with only $(n+1)$ polydisks at each point. The analytical difficulties in doing so are similar to those of studying the Pompeiu problem in the case of symmetric spaces of rank $>1$.

\section{References}

[1] M. Agranovsky, C.A. Berenstein, D.C. Chang and D. Pascuas, Injectivity of the Pompeiu transform in the Heisenberg group, J. Analyse Math., 63 (1994), 131-173. 
[2] M. Agranovsky, C.A. Berenstein and D.C. Chang, Morera theorem for holomorphic $H^{p}$ spaces in the Heisenberg group, J. reine angew. Math., 443 (1993), 49-89.

[3] L. Aizenberg, Carleman's Formulas in Complex Analysis, Kluwer Acad. Publ., Dodrecht, 1993.

[4] L.A. Aizenberg and Sh.A. Dautov, Differential forms orthogonal to holomorphic functions or forms, and their properties, Amer. Math. Soc., Providence, 1983.

[5] L.A. Aizenberg and A.P. Yuzhakov, Integral representations and residues in multidimensional complex analysis, Nauka, Novosibirsk, 1979. English translation, Amer. Math. Soc., Providence, 1983.

[6] C.A. Berenstein and R. Gay, Sur la synthése spectrale dans les espaces symmetriques, J. Math. Pures Appl., 65 (1986), 323-333.

[7] A local version of the 2-circles theorem, Israel J. Math., 55 (1987), 521-544.

[8] L Le probléme de Pompeiu local, J. Analyse Math., 52 (1989), 15-51.

[9] C.A. Berenstein and L. Zalcman, The Pompeiu problem in symmetric spaces, Comment. Math. Helvetici, 55 (1980), 593-621.

[10] L. Brown, B.M. Schreiber and B.A. Taylor, Spectral synthesis and the Pompeiu problem, Ann. Inst. Fourier, 23 (1973), 125-154.

[11] J. Delsarte, Lectures on topics in mean periodic functions and the two-radius theorem, Tata Institute, Bombay, 1961.

[12] P.A. Griffits and J. Harris, Principles of algebraic geometry, John Wiley and Sons, New York, 1978.

[13] L. Zalcman, Offbeat integral geometry, Amer. Math. Monthly, 87 (1980), 161-175.

[14] A bibliographic survey of the Pompeiu problem, Approximation by solutions of partial differential equations, B. Fuglede et al. (eds.), Kluwer Acad. Publ., (1992), 185-194.

Received August 30, 1994. The first author was partially supported by NSF grant DMS9000619. The second author was partially supported by NSF grants DMS-9225043 and CDR-880012.

INSTITUTE FOR SYSTEMS RESEARCH

UNIVERSITY OF MARYLAND

College PARK, MD 20742

E-mail address: carlos@Glue.umd.edu 
Mogens L. Hansen and Richard V. Kadison, Banach algebras with uni-

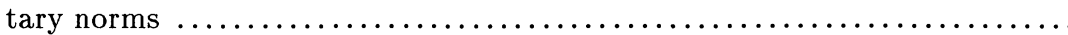

Xin-hou Hua, Sharing values and a problem due to C.C. Yang ..........

Jing-Song Huang, Harmonic analysis on compact polar homogeneous

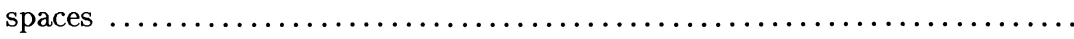

Min-Jei Huang, Commutators and invariant domains for Schrödinger prop-

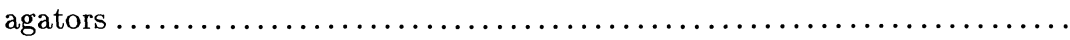

Hisao Kato, Chaos of continuum-wise expansive homeomorphisms and dy-

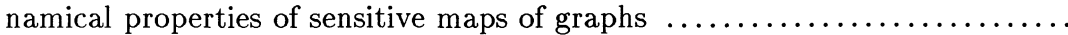

Oliver Küchle, Some properties of Fano manifolds that are zeros of sections in homogeneous vector bundles over Grassmannians ...................

Xin Li and Francisco Marcellan, On polynomials orthogonal with respect to Sobolev inner product on the unit circle $\ldots \ldots \ldots \ldots \ldots \ldots \ldots \ldots \ldots \ldots$. . . . . . .

Steven Liedahl, Maximal subfields of $Q(i)$-division rings .............. 147

Alan L.T. Paterson, Virtual diagonals and $n$-amenability for Banach algebras ....................................................

Claude Schochet, Rational Pontryagin classes, local representations, and

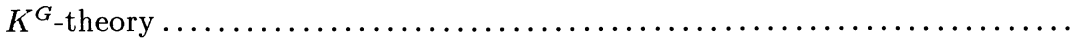

Sandra L. Shields, An equivalence relation for codimension one foliations of 3-manifolds

D. Siegel and E. O. Talvila, Uniqueness for the $n$-dimensional half space

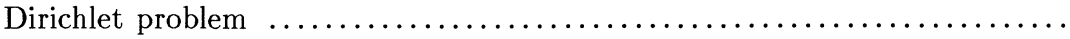

Aleksander Simonič, A Construction of Lomonosov functions and applica-

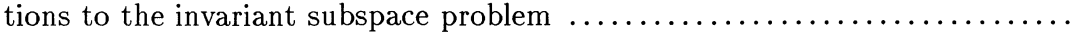

Endre Szabó, Complete intersection subvarieties of general hypersurfaces.. 


\section{PACIFIC JOURNAL OF MATHEMATICS}

Volume $175 \quad$ No. $2 \quad$ October 1996

Mean-value characterization of pluriharmonic and separately harmonic functions

LeV Abramovich AĬZEnberg, CARlos A. Berenstein and L.

WERTHEIM

Convergence for Yamabe metrics of positive scalar curvature with integral bounds on 307 curvature

\section{KAZUO AKUTAGAWA}

Generalized modular symbols and relative Lie algebra cohomology

\section{AVNER DOLNICK ASH and DAVID GINZBURG}

Convolution and limit theorems for conditionally free random variables

MaReK BożejKo, Michael LEINERT and Roland SPEICHER

$L^{p}$-bounds for hypersingular integral operators along curves

SHARAD CHANDARANA

On spectra of simple random walks on one-relator groups. With an appendix by Paul Jolissain

Pierre-Alain Cherix, Alain J. Valette and Paul Jolissaint

Every stationary polyhedral set in $\mathbf{R}^{n}$ is area minimizing under diffeomorphisms

JAIGYOUNG CHOE

Ramanujan's master theorem for symmetric cones

Hongming Ding, Kenneth I. GRoss and Donald Richards

On norms of trigonometric polynomials on SU(2)

AnTHONy H. DOOLEY and SANJiV Kumar GuPTA

On the symmetric square. Unit elements

YUVAL ZVI FLICKER

Stable constant mean curvature surfaces minimize area

KARSTEN GROSSE-BRAUCKMANN

Banach algebras with unitary norms

Mogens LEMVIG HANSEN and RiCHARD VinCENT KADISON

Harmonic analysis on compact polar homogeneous spaces

JING-SONG HUANG

Uniqueness for the $n$-dimensional half space Dirichlet problem

DAVID SIEGEL and ERIK O. TALVILA 